\title{
Numerical estimation of choice of the regularization parameter for NMR $T_{2}$ inversion
}

\author{
You-Long Zou ${ }^{1} \cdot$ Ran-Hong $\mathrm{Xie}^{1} \cdot$ Alon $\mathrm{Arad}^{2}$
}

Received: 5 July 2015/Published online: 20 April 2016

(C) The Author(s) 2016. This article is published with open access at Springerlink.com

\begin{abstract}
Nuclear Magnetic Resonance (NMR) $\mathrm{T}_{2}$ inversion is the basis of NMR logging interpretation. The regularization parameter selection of the penalty term directly influences the NMR $\mathrm{T}_{2}$ inversion result. We implemented both norm smoothing and curvature smoothing methods for NMR $\mathrm{T}_{2}$ inversion, and compared the inversion results with respect to the optimal regularization parameters $\left(\alpha_{\text {opt }}\right)$ which were selected by the discrepancy principle (DP), generalized cross-validation (GCV), S-curve, L-curve, and the slope of L-curve methods, respectively. The numerical results indicate that the DP method can lead to an oscillating or oversmoothed solution which is caused by an inaccurately estimated noise level. The $\alpha_{\mathrm{opt}}$ selected by the L-curve method is occasionally small or large which causes an undersmoothed or oversmoothed $\mathrm{T}_{2}$ distribution. The inversion results from GCV, S-curve and the slope of L-curve methods show satisfying inversion results. The slope of the L-curve method with less computation is more suitable for NMR $\mathrm{T}_{2}$ inversion. The inverted $T_{2}$ distribution from norm smoothing is better than that from curvature smoothing when the noise level is high.
\end{abstract}

You-Long Zou

zoyolo_ok@126.com

Ran-Hong Xie

xieranhong@cup.edu.cn

1 State Key Laboratory of Petroleum Resources and Prospecting, China University of Petroleum, Beijing 102249, China

2 Shell International Exploration and Production Inc., Houston 77079, TX, USA

Edited by Jie Hao
Keywords NMR $\mathrm{T}_{2}$ inversion - Tikhonov regularization · Variable substitution · Levenberg-Marquardt method . Regularization parameter selection

\section{Introduction}

NMR logging directly measures the signal from protons in the fluid in formation of pores. Its applications include fluid typing, porosity calculation, permeability estimation, fluid saturation determination, and bound water estimation. NMR logging interpretation is based on the inverted $T_{2}$ distributions from acquired echo trains. NMR $\mathrm{T}_{2}$ inversion is an ill-posed problem, so it is critical to choose a robust and efficient inversion method to obtain credible NMR spectra. For NMR $\mathrm{T}_{2}$ inversion, scholars have proposed many kinds of inversion methods. Butler, Reeds, and Dawson (BRD) proposed a method to solve norm smoothing with a non-negative constraint of solution (Butler et al. 1981). Dunn et al. (1994) proposed another method for solving norm smoothing with a non-negative constraint of solution. Prammer (1994) used the singular value decomposition (SVD) method for $\mathrm{NMR} \mathrm{T}_{2}$ inversion, and adopted a series of measures to improve the inversion speed for the purpose of real-time processing. Borgia et al. (1998) put forward a complex curvature smoothing method which is called uniform-penalty (UPEN) method, and then made further modifications to the method which allows the regularization parameter to be a variable in the iterative process (Borgia et al. 2000). The SVD method implements the non-negative constraint of solution by singular value truncation which decreases the accuracy of the solution (Prammer 1994; Ge et al. 2016). For the SVD method, the low signal-to-noise ratio of NMR logging data leads to a large cutoff of singular value, which seriously decreases 
the accuracy of the solution. Norm smoothing and curvature smoothing can usually obtain more satisfactory solutions than the SVD method.

The critical issue of norm smoothing and curvature smoothing methods (Dunn et al. 1994) is to determine the optimal regularization parameter. The different optimal regularization parameters selected by different regularization parameter selection methods will cause slightly different inversion results. The published literature mainly used the BRD (Butler et al. 1981) and S-curve (Sezginer 1994; Song et al. 2002) methods to select the regularization parameter for NMR $T_{2}$ inversion. Compared with BRD method, the S-curve method does not need to know the noise level. Except for the above two methods, the generalized cross-validation (GCV) (Golub et al. 1979) and L-curve (Hansen 1992) methods are often widely used to select the regularization parameter for data inversion in many fields. But every regularization parameter selection method has its own advantages and disadvantages. For different inversion problems, we need to comprehensively account for both the amount of calculation and the accuracy of inversion result to determine the most satisfactory parameter selection method for the studied inverse problem. For NMR logging $T_{2}$ inversion, we implement the inversion procedure at each well-logging depth point, so inversion speed should also be an important consideration. This paper implemented norm smoothing and curvature smoothing methods for NMR $\mathrm{T}_{2}$ inversion, and compared the inversion results with respect to the optimal regularization parameters which were selected by the DP, GCV, S-curve, L-curve, and the slope of L-curve methods, respectively.

\section{NMR $T_{2}$ inversion}

The measured echo amplitude of NMR logging using a Carr-Purcell-Meiboom-Gill (CPMG) pulse sequence with sufficient polarization time has the following equation:

$b(t)=\int f\left(\mathrm{~T}_{2}\right) \exp \left(-t / \mathrm{T}_{2}\right) \mathrm{dT}_{2}+\varepsilon$,

where $b(t)$ is the echo amplitude at time $t, \mathrm{~T}_{2}$ is the transverse relaxation time, $\exp \left(-t / \mathrm{T}_{2}\right)$ is the kernel function, $f\left(\mathrm{~T}_{2}\right)$ is the amplitude of $\mathrm{T}_{2}$ distribution, and $\varepsilon$ is noise.

The discrete form of Eq. (1) is

$A_{m \times n} f_{n \times 1}=b_{m \times 1}+\varepsilon_{m \times 1}$,

where $A_{m \times n}$ is kernel matrix, $f_{n \times 1}=\left[f\left(\mathrm{~T}_{2,1}\right), \ldots, f\left(\mathrm{~T}_{2, n}\right)\right]^{\mathrm{T}}$, $b_{m \times 1}=\left[b\left(t_{1}\right), \ldots, b\left(t_{m}\right)\right]^{\mathrm{T}}$.

As is known, NMR $\mathrm{T}_{2}$ inversion is an ill-posed problem, so regularization terms are needed to be added. The most common form of regularization is the Tikhonov regularization, which has the following objective function: $\min \left\{\phi(f)=\frac{1}{2}\|W(A f-b)\|^{2}+\frac{\alpha}{2}\|L f\|^{2}\right\}$,

where $\|\cdot\|$ means Euclidean norm and $W$ is a weighted matrix whose diagonal elements equal to the reciprocal of the noise level. If the noise level of data is a constant, $W$ can be an identity matrix. $L$ is the regularization matrix, can be a zero-, or first-, or second-derivative operator which corresponds to norm smoothing, slope smoothing, and curvature smoothing (Dunn et al. 1994). $\alpha$ is the regularization parameter.

To obtain the non-negative constraint of solution, the iterative solution is commonly made by eliminating the columns of kernel matrix corresponding to the negative components in the solution or replacing them with large constants. Unlike the above-mentioned methods, we use a variable substitution method to obtain a non-negative constraint of solution, in which the solution is substituted by a non-negative expression. For example, $\operatorname{set} f=\exp (x)$ or $x^{2}$, the above objective function of Eq. (3) can be rewritten as

$\min \left\{\phi(x)=\frac{1}{2}\|W(A \exp (x)-b)\|^{2}+\frac{\alpha}{2}\|L \exp (x)\|^{2}\right\}$,

or

$\min \left\{\phi(x)=\frac{1}{2}\left\|W\left(A x^{2}-b\right)\right\|^{2}+\frac{\alpha}{2}\left\|L x^{2}\right\|^{2}\right\}$.

The objective functions of Eqs. (4a) and (4b) without constraint conditions can be solved by the LevenbergMarquardt method, an iterative method. The new solution $x_{\text {new }}$ is updated by the following equations (Madsen and Nielsen 2010):

$\left(\phi^{\prime \prime}+\mu I\right) \Delta x=-\phi^{\prime}$,

$x_{\text {new }}=x_{\text {old }}+\Delta x$,

where $I$ is an identity matrix, $\phi^{\prime}$ is the gradient of objective function, $\phi^{\prime \prime}$ is the Hessian matrix of objective function, $\mu$ is a parameter that can be updated in every iteration by the updating strategy of Madsen and Nielsen (Madsen and Nielsen 2010).

By calculating the partial derivatives of the objective functions of Eqs. (4a) and (4b) with respect to $x$, we can obtain the gradients $\phi^{\prime}$ and approximate symmetric positive definite Hessian matrices $\phi^{\prime \prime}$ of the objective functions of Eqs. (4a) and (4b), respectively.

For $f=\exp (x)$,

$$
\begin{aligned}
\phi^{\prime}= & (W \cdot A \cdot \operatorname{diag}(\exp (x)))^{\mathrm{T}}(W \cdot A \cdot \exp (x)-W \cdot b) \\
& +\alpha(L \cdot \operatorname{diag}(\exp (x)))^{\mathrm{T}}(L \cdot \exp (x)), \\
\phi^{\prime \prime} \approx & (W \cdot A \cdot \operatorname{diag}(\exp (x)))^{\mathrm{T}}(W \cdot A \cdot \operatorname{diag}(\exp (x))) \\
& +\alpha(L \cdot \operatorname{diag}(\exp (x)))^{\mathrm{T}}(L \cdot \operatorname{diag}(\exp (x))) .
\end{aligned}
$$




$$
\begin{aligned}
& \text { For } f=x^{2}, \\
& \phi^{\prime}= 2.0 \cdot(W \cdot A \cdot \operatorname{diag}(x))^{\mathrm{T}}\left(W \cdot A \cdot x^{2}-W \cdot b\right)+2.0 \\
& \cdot \alpha(L \cdot \operatorname{diag}(x))^{\mathrm{T}}\left(L \cdot x^{2}\right),
\end{aligned}
$$$$
\phi^{\prime \prime} \approx 4.0 \cdot(W \cdot A \cdot \operatorname{diag}(x))^{\mathrm{T}}(W \cdot A \cdot \operatorname{diag}(x))+4.0
$$$$
\cdot \alpha(L \cdot \operatorname{diag}(x))^{\mathrm{T}}(L \cdot \operatorname{diag}(x)),
$$

where $\operatorname{diag}(\exp (x))$ and $\operatorname{diag}(x)$ are diagonal matrices which are generated by vectors $\exp (x)$ and $x$, respectively.

\section{Regularization parameter selection}

Since a too small or too large regularization parameter can result in an undersmoothed or oversmoothed solution, it is critical to choose an optimal regularization parameter $\left(\alpha_{\text {opt }}\right)$. The commonly used regularization parameter selection methods include the DP, GCV, S-curve, and L-curve methods (Morozov 1966; Golub et al. 1979; Sezginer 1994; Hansen 1992).

\subsection{Discrepancy principle (DP)}

If the noise level $\sigma$ is known, the DP (Morozov 1966) suggests that the $\alpha_{\mathrm{opt}}$ should be chosen to satisfy the following equation:

$\zeta(\alpha)=\|A f-b\|^{2}=\tau m \sigma^{2}$,

where $m$ is the number of the echoes of the echo train b. $\tau \geq 1$ is a predetermined real number, typically $\tau=1$.

Figure 1 shows the typical "S" shape curves of the variation of regularization parameter $(\alpha)$ with residual norm $(\zeta(\alpha))$ for norm smoothing and curvature smoothing. This method needs to know the noise level $\sigma$, but sometimes it is difficult to estimate an accurate $\sigma$.

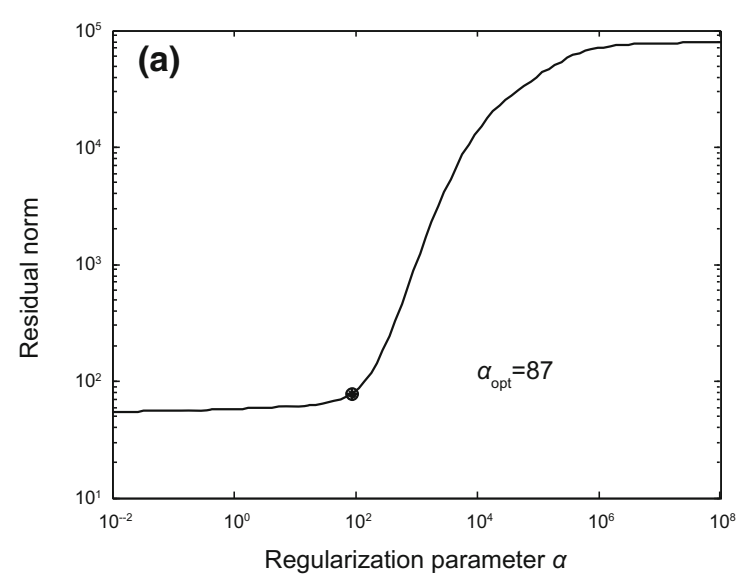

\subsection{Generalized cross-validation (GCV)}

The GCV method was proposed by Golub et al. (1979) to find the $\alpha_{\mathrm{opt}}$ that minimizes the GCV function. For Tikhonov regularization, the GCV function is

$G(\alpha)=\frac{\left\|b-A f_{\alpha}\right\|^{2}}{\operatorname{trace}\left(I-A A^{\#}\right)^{2}}$,

where $I$ is an identity matrix, $A^{\#}$ denotes the regularized pseudo-inverse of $A, f_{\alpha}=A^{\#} b$. For Tikhonov regularization, $A^{\#}=\left(A^{\mathrm{T}} A+\alpha L^{\mathrm{T}} L\right)^{-1} A^{\mathrm{T}}$.

Figure 2 shows the variation of the GCV function value with a regularization parameter $(\alpha)$ for norm smoothing and curvature smoothing. As shown in Fig. 2, as $\alpha$ increases, the GCV function value first decreases and then increases.

\subsection{S-curve}

The S-curve method (Sezginer 1994; Song et al. 2002) finds the minimum $\alpha$ as $\alpha_{\text {opt }}$ that satisfies

$\frac{\mathrm{d} \log \zeta}{2 \mathrm{~d} \log \alpha}=\mathrm{tol}$,

where $0<t o l<1$ is a predetermined constant, typically $t o l=0.1$.

This method uses the slope of the S-curve as the criterion of $\alpha_{\text {opt }}$ selection. Eq. (21) in Appendix shows the specific formula of the slope of S-curve. The heel of the S-curve is selected as $\alpha_{\text {opt }}$, which balances the residual and known noise variance (Song et al. 2002).

Figure 3 shows the variation of slope of S-curve with regularization parameter $(\alpha)$. Typically, the slope of the S-curve gradually increases at first, then remains at a large value, and finally gradually decreases. We choose the smallest $\alpha$ that satisfies the Eq. (11) as $\alpha_{\text {opt }}$.

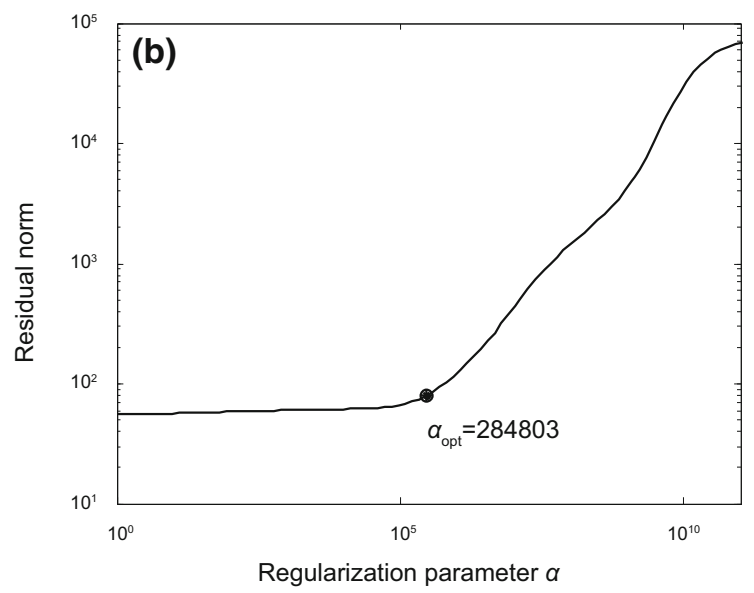

Fig. 1 The variation of residual norm with regularization parameter. a Norm smoothing, b curvature smoothing 

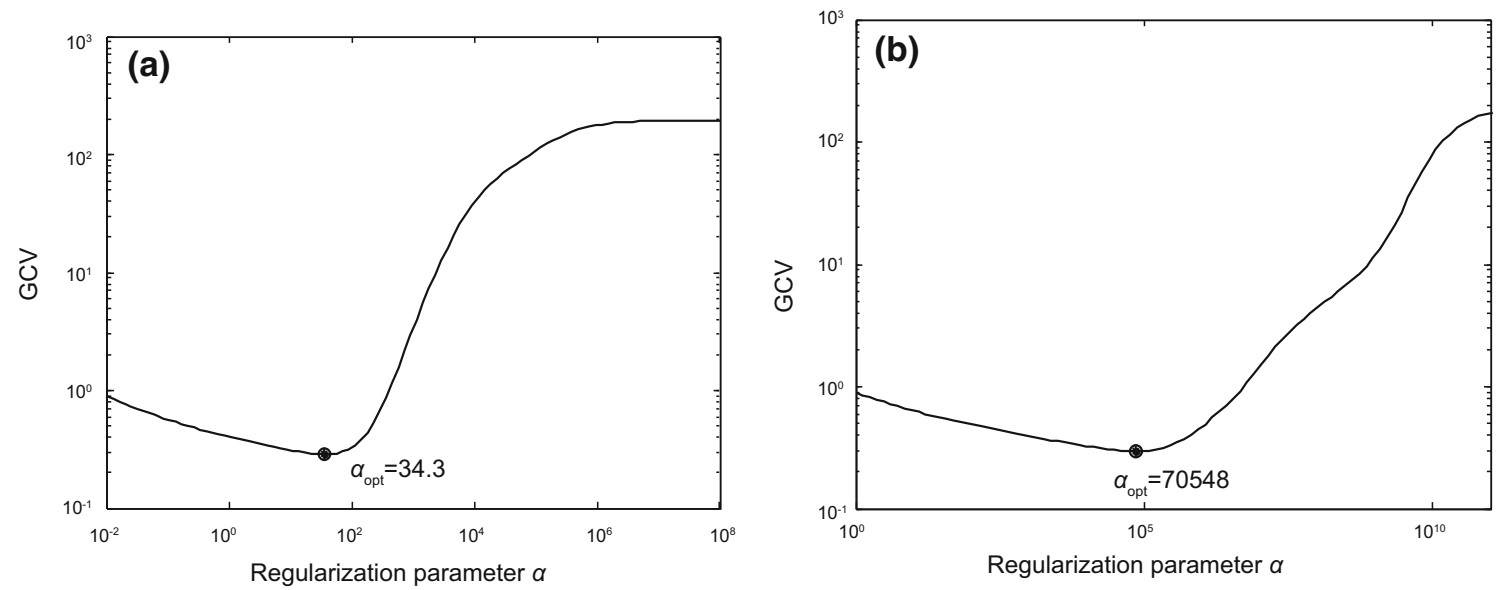

Fig. 2 The variation of GCV function value with regularization parameter. a Norm smoothing, b curvature smoothing
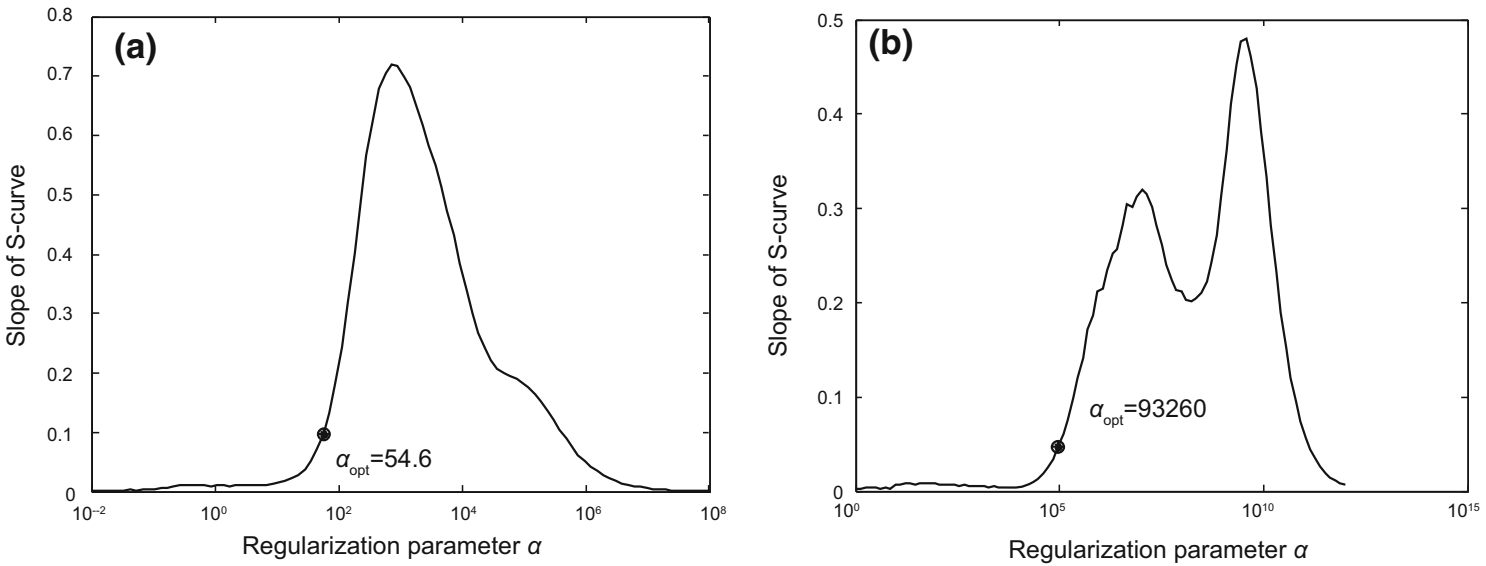

Fig. 3 The variation of slope of the S-curve with regularization parameter. a Norm smoothing, $\mathbf{b}$ curvature smoothing

\subsection{L-curve}

As $\alpha$ increases, solution norm $\eta(\alpha)=\|L f\|^{2}$ decreases, while residual norm $\zeta(\alpha)$ increases. In the $\log -\log$ scale, the curve formed by $\eta(\alpha)$ versus $\zeta(\alpha)$ for each of a set of $\alpha$ has an " $L$ " shape, so it is called the L-curve. The method was proposed by Lawson and popularized by Hansen (Hansen 1992).

Figure 4 shows the typical L-curves of norm smoothing and curvature smoothing. Intuitively, $\alpha_{\text {opt }}$ should lie on the "corner" of the L-curve, for values higher than this "corner", $\zeta(\alpha)$ increases without reducing $\eta(\alpha)$ too much, while for values smaller than this "corner", $\zeta(\alpha)$ decreases little but with a rapid increase of $\eta(\alpha)$.

People have proposed many kinds of methods to locate the "corner" (the point of maximum curvature) of the L-curve. Castellanos et al. (2002) analyzed the drawbacks of three methods for finding the corner of L-curve (Kaufman and Neumaier 1996; Hansen 1998; Guerra and Hernandez 2001), and proposed a robust triangle method. Hansen et al.
(2007) proposed an adaptive pruning algorithm, which first calculates the corner candidates at different scales or resolutions and then selects the overall optimal corner from the candidates. The above-mentioned methods are indirect methods to calculate the corner of L-curve. However, indirect methods cannot guarantee correct results in all cases. This article gives an Eq. (27) for directly calculating the curvature of the L-curve of single-parameter Tikhonov regularization, and the specific derivation is shown in Appendix. Figure 5 shows the variation of the curvature of L-curve with regularization parameter $(\alpha)$.

\subsection{The slope of the L-curve}

Comparing the S-curve (Fig. 1) with the L-curve (Fig. 4), we can see that the two types of curves have similar shapes. If we exchange the horizontal and vertical coordinates of the L-curve, the resulting curve is the mirror image of the $\mathrm{S}$-curve. It naturally refers us to the S-curve method, so we 

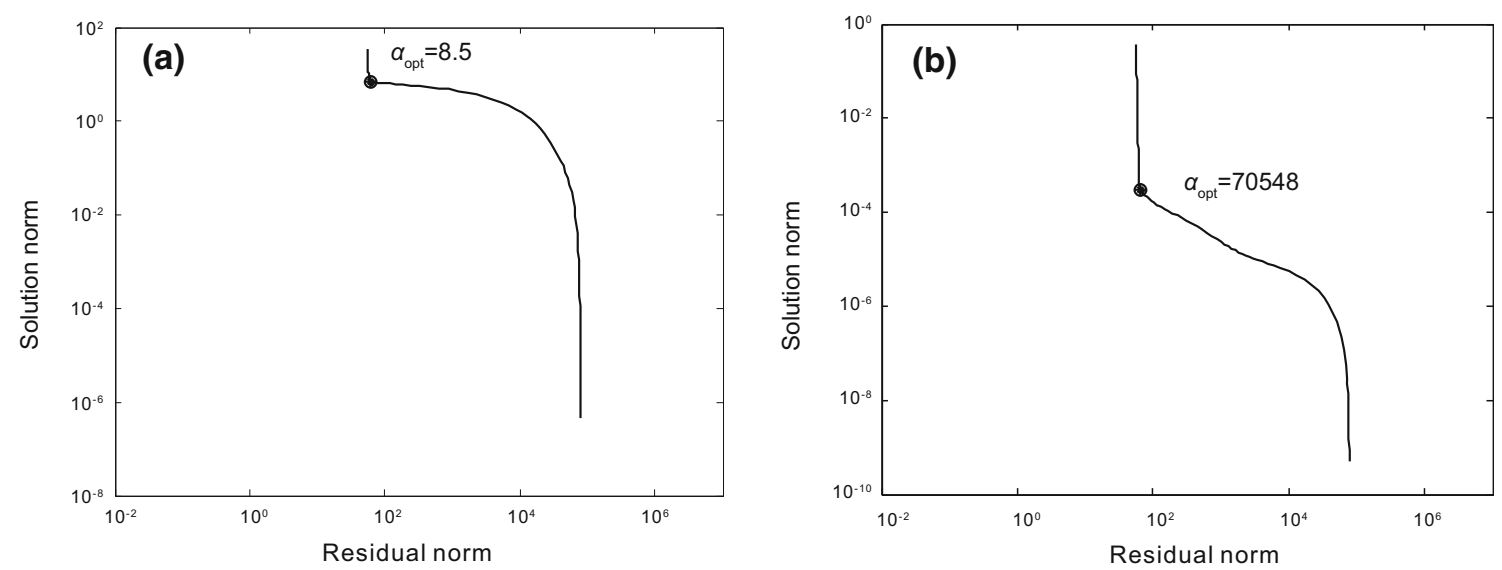

Fig. 4 L-curve. a Norm smoothing, b curvature smoothing
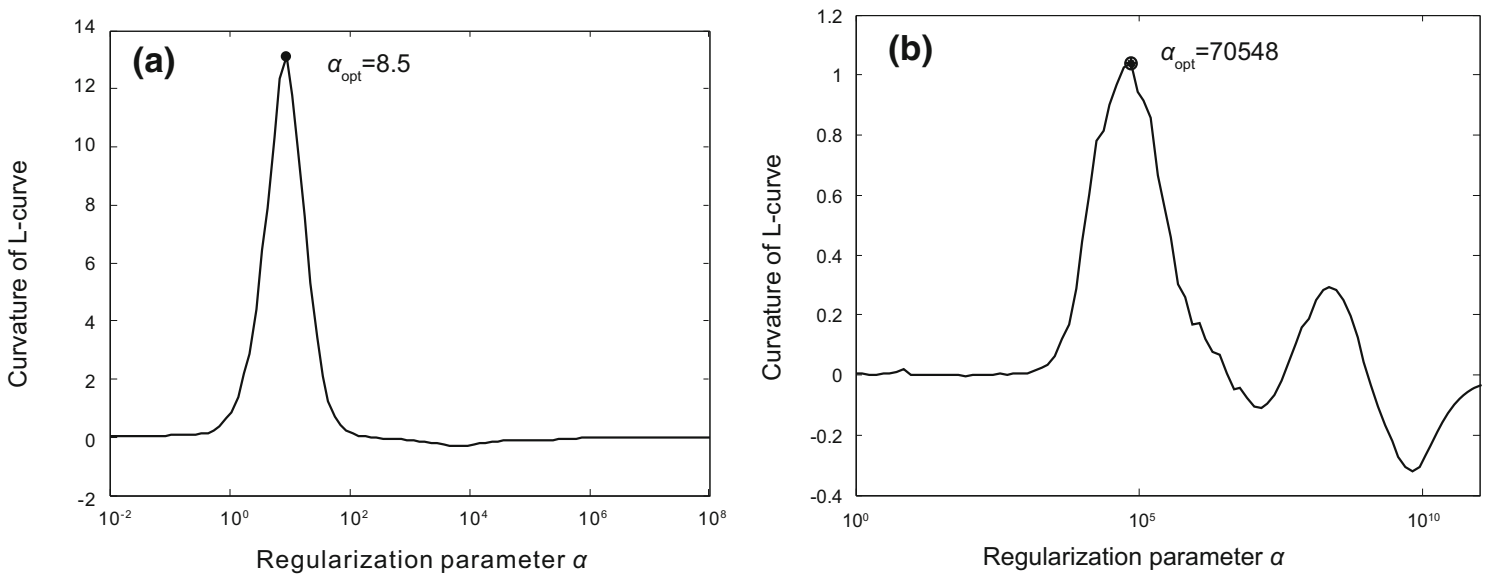

Fig. 5 The variation of the curvature of L-curve with regularization parameter. a Norm smoothing, b curvature smoothing
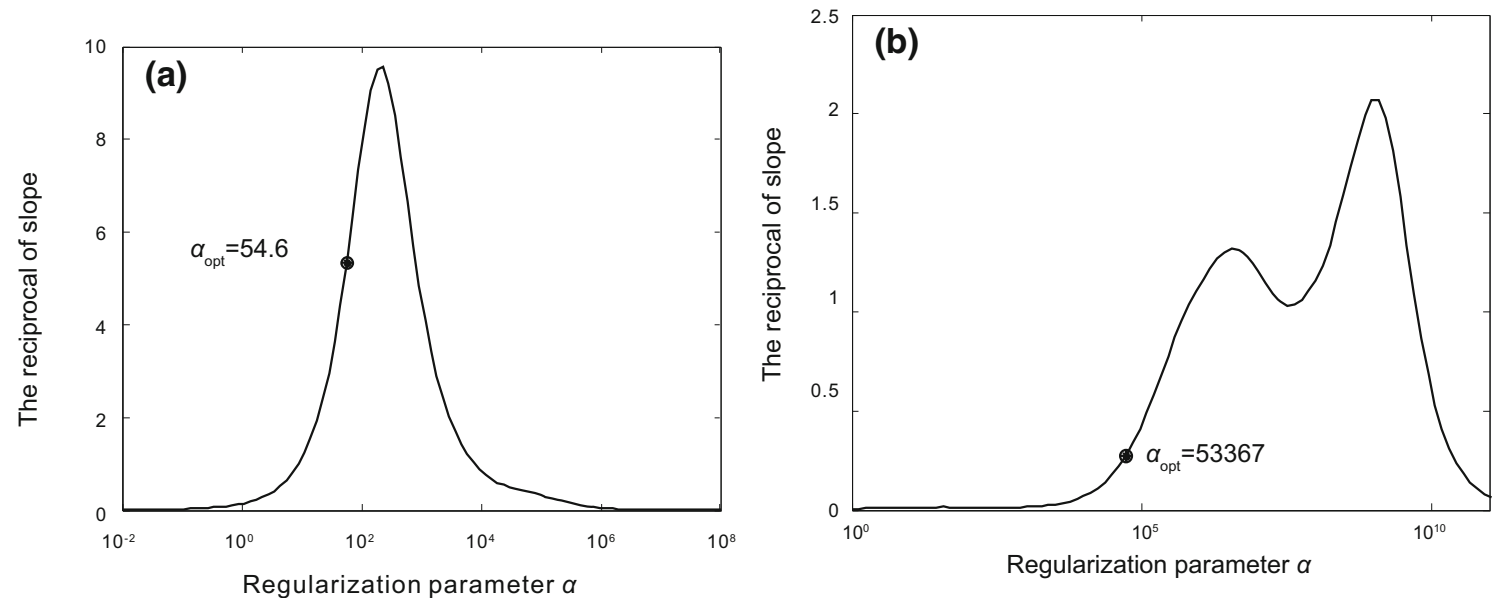

Fig. 6 The variation of the reciprocal of the absolute value of the slope of L-curve with regularization parameter. a Norm smoothing, b curvature smoothing

can use the slope of the L-curve to select the regularization parameter. According to Eq. (24), the slope of the L-curve has a simple formula and can avoid the matrix inversion of the S-curve method (see Eq. (21)). Figure 6 shows the variation of the reciprocal of the absolute value of the slope of the L-curve with regularization parameter $(\alpha)$. 
Comparing Fig. 6 with Fig. 3, it can be found that the two types of curves have striking similarities. So, we attempt to use the slope of the L-curve criterion to select the regularization parameter, and compare its results with those of the other methods. The threshold of the reciprocal of the absolute value of the slope of the L-curve can be selected in the interval $[0.1,10]$. Here we choose the threshold values of 5 and 0.25 for norm smoothing and curvature smoothing methods, respectively.

\section{Numerical results}

A bimodal $\mathrm{T}_{2}$ distribution model is constructed as shown in Fig. 7, where $64 T_{2}$ components are preselected between 0.1 and $10,000 \mathrm{~ms}$. Using this $\mathrm{T}_{2}$ distribution model, we generated echo trains with 500 echoes, where echo spacing is $0.9 \mathrm{~ms}$. Different level Gaussian random noise was applied, as shown in Fig. 8. The red line represents the echo train without noise, and the green, blue, magenta, and black lines show the echo trains with noise levels of 0.25 , $0.5,1.0$, and 2.0 porosity unit (pu), respectively.

To improve the inversion speed of NMR echo data, the echo trains are usually compressed before inversion (Sezginer 1994; Dunn and LaTorraca 1999; Venkataramanan et al. 2002; Zou and Xie 2015). Here, we compress the NMR echo data with the SVD method (Sezginer 1994; Zou and Xie 2015). After compression, echoes in each echo train shown in Fig. 8 are all compressed to 20 data points for NMR $\mathrm{T}_{2}$ inversion.

We select optimal regularization parameters $\left(\alpha_{\text {opt }}\right)$ by the DP, GCV, S-curve, L-curve, and the slope of L-curve methods, respectively, and compare the NMR $\mathrm{T}_{2}$ inversion results from different regularization parameter selection methods. Figure 9 shows the NMR $\mathrm{T}_{2}$ inversion results of

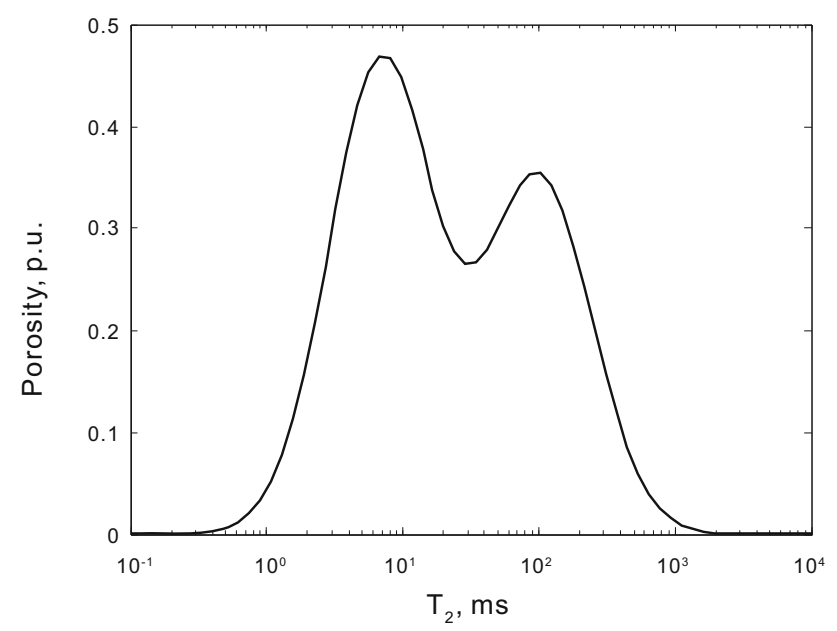

Fig. 7 The $T_{2}$ distribution model

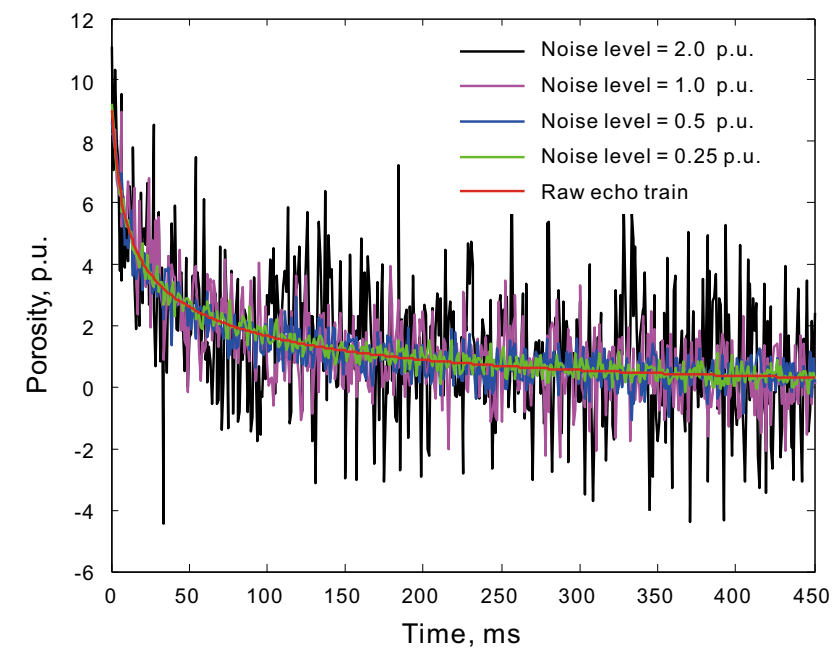

Fig. 8 The computed echo trains from $T_{2}$ distribution model with different noise levels

norm smoothing and curvature smoothing methods with respect to noise levels of $0.25,0.5,1.0$, and $2.0 \mathrm{pu}$. The black line is the $\mathrm{T}_{2}$ distribution model, and the green, blue, magenta, red, and cyan lines are the $\mathrm{T}_{2}$ distribution inversion results according to the DP, GCV, S-curve, L-curve, and the slope of L-curve methods, respectively. As shown in Fig. 9, the selected $\alpha_{\text {opt }}$ from the DP method is sometimes small or large (because of the underestimated or overestimated echo data noise level) that leads to an undersmoothed or oversmoothed solution. The $\alpha_{\text {opt }}$ selected by L-curve method is occasionally small or large which leads to an undersmoothed or oversmoothed $\mathrm{T}_{2}$ distribution. The inversion results from the GCV, S-curve, and the slope of L-curve methods are close and satisfactory. Curvature smoothing can better suppress the oscillation caused by noise than norm smoothing, and can obtain a smoother solution than norm smoothing. The curvature smoothing makes the inverted $T_{2}$ distribution prone to show single peak shape than norm smoothing when the noise level is high. Table 1 shows the porosity errors of different regularization parameter selection methods, and finds that the DP method occasionally obtains a large porosity error, and the porosity errors of the GCV, S-curve, L-curve, and slope of L-curve methods are close.

\section{Well data processing results}

Well A is in a tight sandstone reservoir with low porosity. The signal-to-noise ratio (SNR) of the NMR logging data is low. Figure 10 shows the inverted $\mathrm{T}_{2}$ distributions of norm smoothing, where the fourth track represents the inverted $\mathrm{T}_{2}$ distributions of the SVD method, the fifth to ninth tracks represent the inversion results of the DP, GCV, S-curve, 

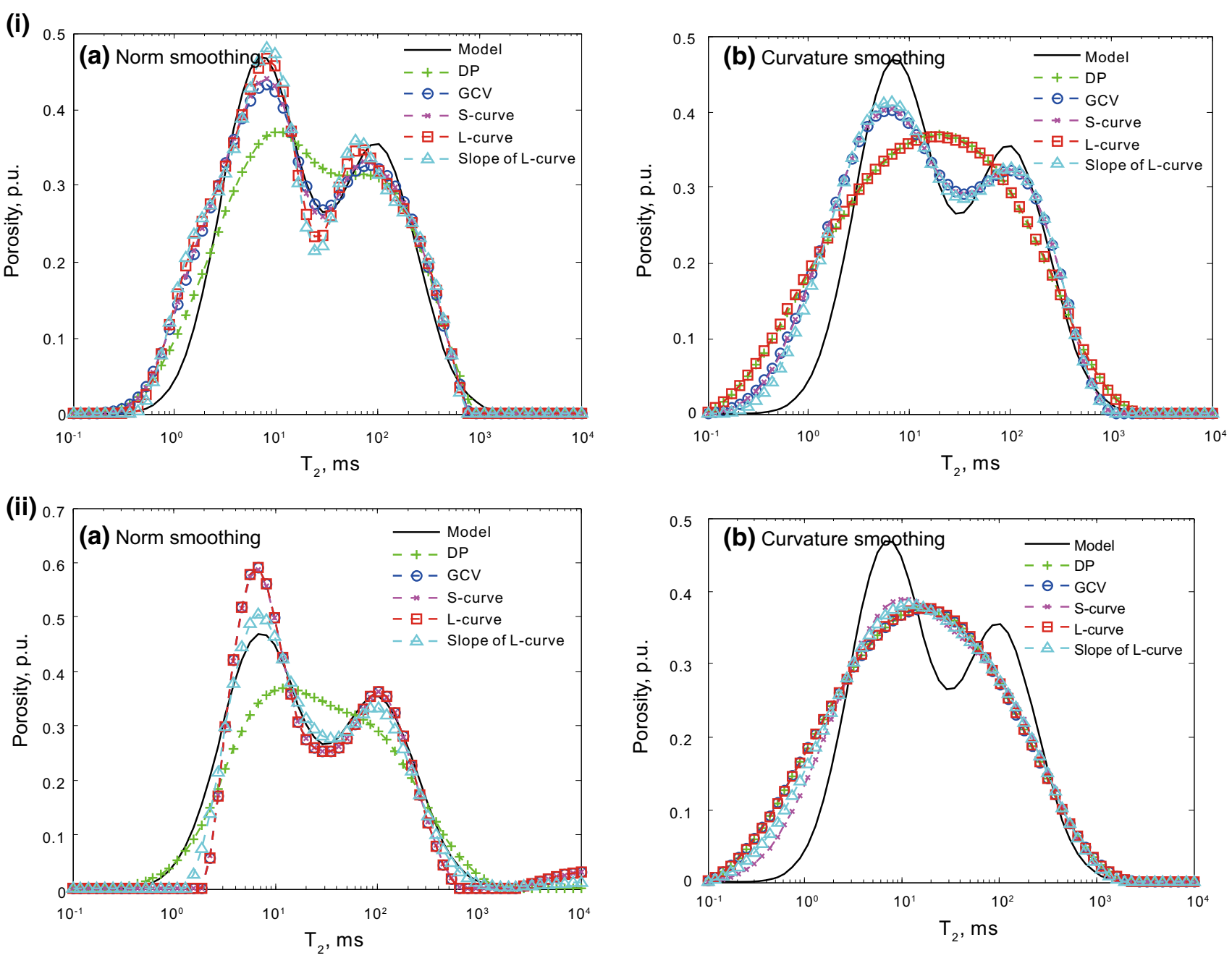

Fig. 9 The inversion results from different regularization parameter selection methods. i Noise level is 0.25 pu. a norm smoothing; b curvature smoothing. ii Noise level is $0.5 \mathrm{pu}$. a norm smoothing; b curvature smoothing. iii Noise level is $1.0 \mathrm{pu}$. a norm smoothing; b curvature smoothing. iv Noise level is $2.0 \mathrm{pu}$. a norm smoothing; $\mathbf{b}$ curvature smoothing

L-curve, and slope of L-curve methods, respectively. As shown in Fig. 10, the inverted $\mathrm{T}_{2}$ distributions of the SVD method, usually with single peak shapes, are oversmoothed. The inverted $\mathrm{T}_{2}$ distributions of norm smoothing are satisfactory. The inverted $\mathrm{T}_{2}$ distributions from the DP and L-curve methods are slightly oversmoothed. However, the inverted $T_{2}$ distributions from the GCV and S-curve methods are slightly undersmoothed. The inverted $T_{2}$ distributions from the slope of L-curve method are relatively more satisfactory than those from other methods.

\section{Conclusions}

This paper uses the Tikhonov regularization with a nonnegative constraint of solution for NMR $\mathrm{T}_{2}$ inversion. The non-negative constraint of solution is implemented by variable substitution, and then the modified objective function is solved by the Levenberg-Marquardt method. The optimal regularization parameters $\left(\alpha_{\mathrm{opt}}\right)$ from norm smoothing and curvature smoothing methods are selected by the DP, GCV, S-curve, L-curve, and the slope of L-curve methods, respectively. The following conclusions are obtained.

(1) The inverted NMR $T_{2}$ distributions from the DP method depend on the estimated noise level which is difficult to estimate accurately. The inversion results from the GCV, S-curve, and the slope of L-curve methods are satisfactory. The small or large $\alpha_{\mathrm{opt}}$ selected by the L-curve method leads to an oscillation or oversmoothed $T_{2}$ distribution. When the noise level is high, norm smoothing can more effectively than curvature smoothing avoid the bimodal $T_{2}$ distribution being converted to a single peak distribution. The inverted $T_{2}$ distribution of 

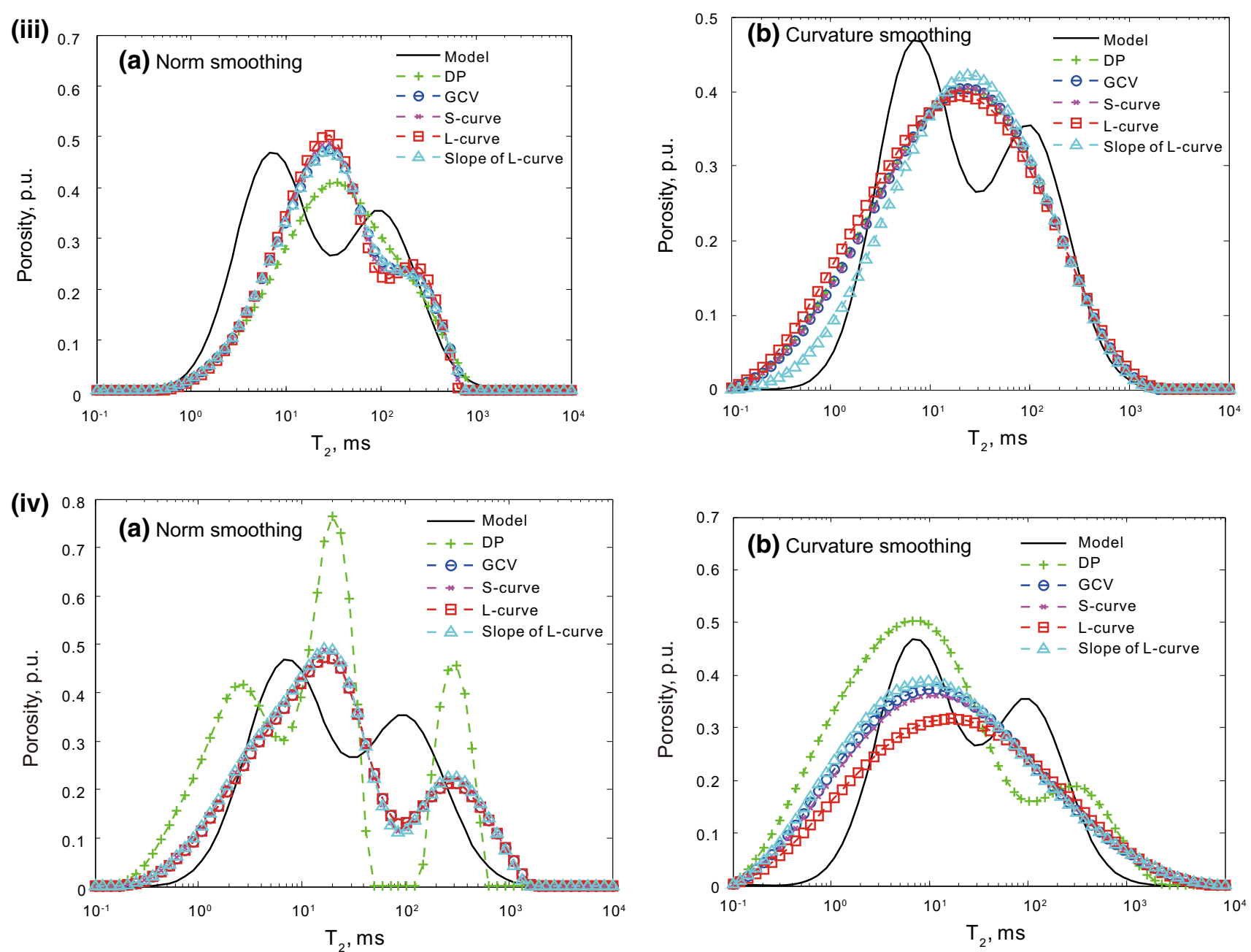

Fig. 9 continued

Table 1 Porosity errors obtained from different regularization parameter selection methods (unit: pu)

\begin{tabular}{llrrrr}
\hline Method & DP & \multicolumn{1}{l}{ GCV } & \multicolumn{1}{l}{ S-curve } & L-curve & Slope of L-curve \\
\hline Norm smoothing & & & & & \\
Noise $=0.25$ & 0.1835 & -0.5536 & -0.5489 & -0.5857 & -0.5858 \\
Noise $=0.5$ & 0.9424 & 0.5039 & 0.5047 & 0.5017 & 0.5295 \\
Noise $=1.0$ & 1.7092 & 1.1347 & 1.1220 & 1.0929 & 1.1759 \\
Noise $=2.0$ & -2.2772 & -0.1316 & -0.3117 & 0.2869 & -0.3521 \\
Curvature smoothing & & & & & \\
Noise $=0.25$ & -1.0283 & -1.1421 & -1.0202 & -1.0802 & -0.8769 \\
Noise $=0.5$ & -0.8131 & -0.8078 & -0.4299 & -0.8134 & -0.6150 \\
Noise $=1.0$ & -0.6772 & -0.5187 & -0.6139 & -0.9139 & 0.0128 \\
Noise $=2.0$ & -3.6340 & -1.1841 & -1.0550 & 0.0955 & -1.6763 \\
\hline
\end{tabular}

norm smoothing is better than that of curvature smoothing.

(2) The GCV and L-curve methods need to calculate the solution of the regularization parameter over a wide range, which needs a large amount of calculation.
The S-curve and the slope of L-curve methods can quickly find the $\alpha_{\text {opt }}$ by iteration, suitable for norm smoothing and curvature smoothing. The slope of L-curve method needs less calculation than the S-curve method does, but the $\mathrm{T}_{2}$ inversion results 


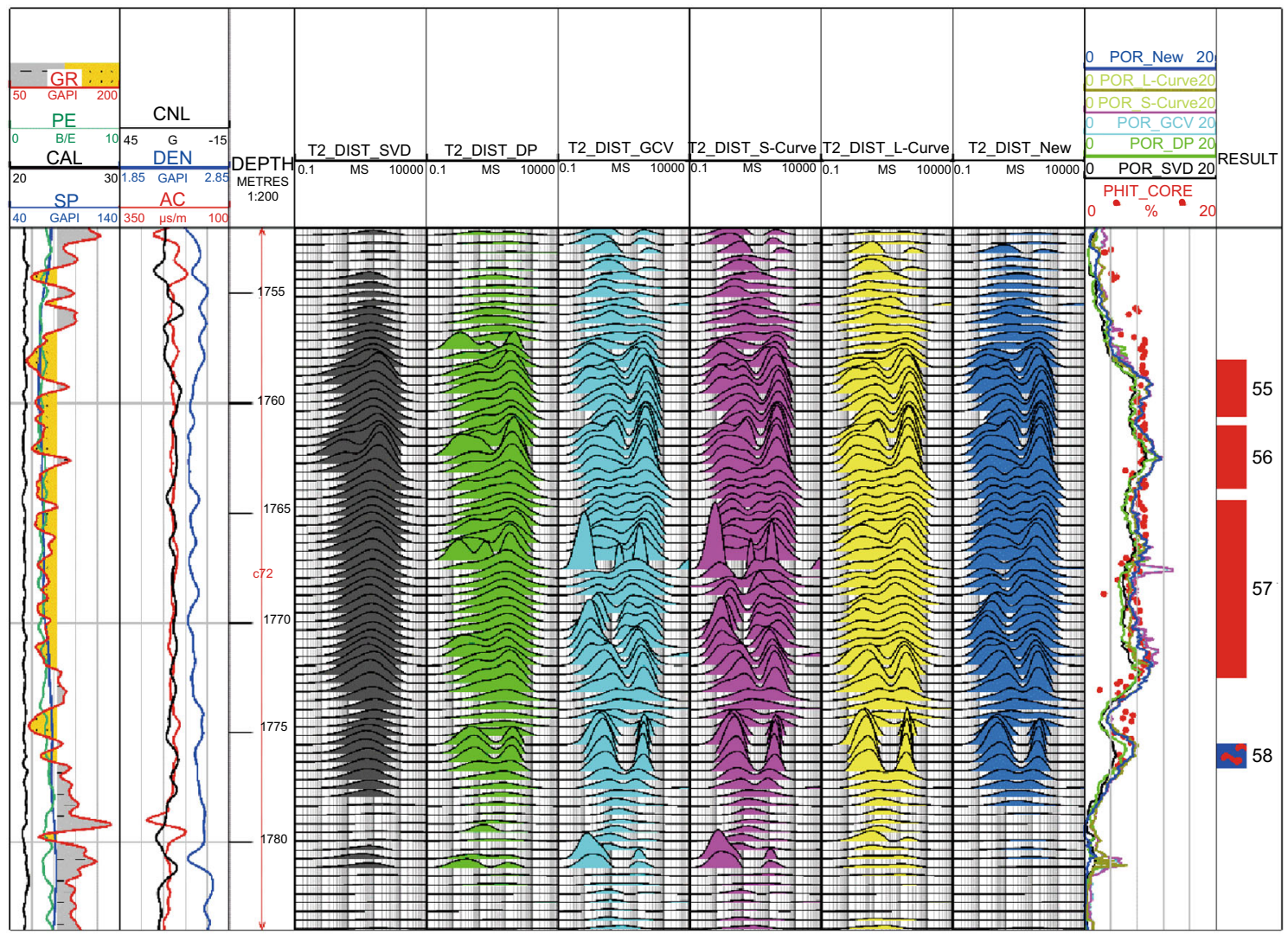

Fig. 10 The inversion results of NMR logging data

from these two methods are close. So, the slope of the L-curve method can be an efficient alternative to the S-curve method.

Acknowledgments This work was funded by Shell International Exploration and Production Inc. (PT45371), the National Natural Science Foundation of China-China National Petroleum Corporation Petrochemical Engineering United Fund (U1262114), and the National Natural Science Foundation of China (41272163).

Open Access This article is distributed under the terms of the Creative Commons Attribution 4.0 International License (http://crea tivecommons.org/licenses/by/4.0/), which permits unrestricted use, distribution, and reproduction in any medium, provided you give appropriate credit to the original author(s) and the source, provide a link to the Creative Commons license, and indicate if changes were made.

\section{Appendix}

The objective function of single-parameter Tikhonov regularization is given by

$\min \left\{\frac{1}{2}\|W(A f-b)\|^{2}+\frac{\alpha}{2}\|L f\|^{2}\right\}$.
The derivative of the above equation with respect to $f$ is written as

$\left((W A)^{\mathrm{T}}(W A)+\alpha L^{\mathrm{T}} L\right) f=(W A)^{\mathrm{T}} W b$.

Let $\zeta=\|W(A f-b)\|^{2}, \eta=\|L f\|^{2}$. The curvature $\kappa$ of Lcurve $(\log \zeta, \log \eta)$ is

$\kappa=\frac{\frac{\mathrm{d}^{2} \log \eta}{\mathrm{d}(\log \zeta)^{2}}}{\left(1+\left(\frac{\mathrm{d} \log \eta}{\mathrm{d} \log \zeta}\right)^{2}\right)^{3 / 2}}$.

To calculate the $\kappa$, we need to compute $\frac{\mathrm{d}^{2} \log \eta}{\mathrm{d}(\log \zeta)^{2}}$ and $\frac{\mathrm{d} \log \eta}{\mathrm{d} \log \zeta}$. Since

$\frac{\mathrm{d} \log \eta}{\mathrm{d} \log \zeta}=\frac{\zeta}{\eta} \frac{\mathrm{d} \eta}{\mathrm{d} \zeta}$

$\frac{\mathrm{d}^{2} \log \eta}{\mathrm{d}(\log \zeta)^{2}}=\frac{\zeta}{\eta} \frac{\mathrm{d} \eta}{\mathrm{d} \zeta}-\frac{\zeta^{2}}{\eta^{2}}\left(\frac{\mathrm{d} \eta}{\mathrm{d} \zeta}\right)^{2}+\frac{\zeta^{2}}{\eta} \frac{\mathrm{d}^{2} \eta}{\mathrm{d} \zeta^{2}}$

the computation of Eq. (14) is converted to compute $\frac{\mathrm{d} \eta}{\mathrm{d} \zeta}$ and $\frac{\mathrm{d}^{2} \eta}{\mathrm{d} \zeta^{2}}$.

The derivative of Eq. (13) with respect to $\alpha$ is 


$$
L^{\mathrm{T}} L f+\left((W A)^{\mathrm{T}}(W A)+\alpha L^{\mathrm{T}} L\right) \frac{\mathrm{d} f}{\mathrm{~d} \alpha}=0 .
$$

So,

$\frac{\mathrm{d} f}{\mathrm{~d} \alpha}=-\left((W A)^{\mathrm{T}}(W A)+\alpha L^{\mathrm{T}} L\right)^{-1} L^{\mathrm{T}} L f$.

We should note that according to the non-negative constraint, only the rows and columns of the matrices $(W A)^{\mathrm{T}}(W A)$ and $L^{\mathrm{T}} L$ corresponding to $f>0$ will be involved in the calculation.

So, the derivative of $\eta$ with respect to $\alpha$ is

$\frac{\mathrm{d} \eta}{\mathrm{d} \alpha}=-2 f^{\mathrm{T}} L^{\mathrm{T}} L\left((W A)^{\mathrm{T}}(W A)+\alpha L^{\mathrm{T}} L\right)^{-1} L^{\mathrm{T}} L f$.

The derivative of $\zeta$ with respect to $\alpha$ is

$\frac{\mathrm{d} \zeta}{\mathrm{d} \alpha}=2 \alpha f^{\mathrm{T}} L^{\mathrm{T}} L\left((W A)^{\mathrm{T}}(W A)+\alpha L^{\mathrm{T}} L\right)^{-1} L^{\mathrm{T}} L f$.

So,

$\frac{\mathrm{d} \log \zeta}{\mathrm{d} \log \alpha}=\frac{2 \alpha^{2} f^{\mathrm{T}} L^{\mathrm{T}} L\left((W A)^{\mathrm{T}}(W A)+\alpha L^{\mathrm{T}} L\right)^{-1} L^{\mathrm{T}} L f}{\zeta}$.

And,

$\frac{\mathrm{d} \eta}{\mathrm{d} \zeta}=\frac{\mathrm{d} \eta}{\mathrm{d} \alpha} \frac{\mathrm{d} \alpha}{\mathrm{d} \zeta}=\frac{-1}{\alpha}$

$\frac{\mathrm{d}^{2} \eta}{\mathrm{d} \zeta^{2}}=\frac{\mathrm{d}\left(\frac{\mathrm{d} \eta}{\mathrm{d} \zeta}\right)}{\mathrm{d} \zeta}=\frac{\mathrm{d}\left(-\frac{1}{\alpha}\right)}{\mathrm{d} \zeta}=\frac{1}{\alpha^{2}} \frac{\mathrm{d} \alpha}{\mathrm{d} \zeta}$.

Substituting Eqs. (22) and (23) into Eqs. (15) and (16), then

$\frac{\mathrm{d} \log \eta}{\mathrm{d} \log \zeta}=\frac{-\zeta}{\alpha \eta}$

$$
\begin{aligned}
\frac{\mathrm{d}^{2} \log \eta}{\mathrm{d}(\log \zeta)^{2}}= & -\frac{\zeta}{\alpha \eta}-\frac{\zeta^{2}}{\alpha^{2} \eta^{2}} \\
& +\frac{\zeta^{2}}{2 \alpha^{3} \eta} \frac{1}{f^{\mathrm{T}} L^{\mathrm{T}} L\left((W A)^{\mathrm{T}}(W A)+\alpha L^{\mathrm{T}} L\right)^{-1} L^{\mathrm{T}} L f} .
\end{aligned}
$$

Substituting Eqs. (21) and (24) into Eq. (25), then

$\frac{\mathrm{d}^{2} \log \eta}{\mathrm{d}(\log \zeta)^{2}}=\frac{\mathrm{d} \log \eta}{\mathrm{d} \log \zeta}-\left(\frac{\mathrm{d} \log \eta}{\mathrm{d} \log \zeta}\right)^{2}-\frac{\mathrm{d} \log \alpha}{\mathrm{d} \log \zeta} \frac{\mathrm{d} \log \eta}{\mathrm{d} \log \zeta}$.

Substituting Eq. (26) into Eq. (14), then

$\kappa=\frac{\frac{\mathrm{d} \log \eta}{\mathrm{d} \log \zeta}-\left(\frac{\mathrm{d} \log \eta}{\mathrm{d} \log \zeta}\right)^{2}-\frac{\mathrm{d} \log \alpha}{\mathrm{d} \log \zeta} \mathrm{d} \log \eta}{\mathrm{d} \log \zeta}$.

\section{References}

Borgia GC, Brown RJS, Fantazzini P. Uniform-penalty inversion of multiexponential decay data. J Magn Reson. 1998;132(1):65-77.

Borgia GC, Brown RJS, Fantazzini P. Uniform-penalty inversion of multiexponential decay data II. Data spacing, $\mathrm{T}_{2}$ data, systematic data errors, and diagnostics. J Magn Reson. 2000;147(2):273-85.

Butler JP, Reeds JA, Dawson SV. Estimating solutions of first kind integral equations with nonnegative constraints and optimal smoothing. SIAM J Numer Anal. 1981;18(3):381-97.

Castellanos JL, Gómez S, Guerra V. The triangle method for finding the corner of the L-curve. Appl Numer Math. 2002;43(4):359-73.

Dunn KJ, LaTorraca GA. The inversion of NMR log data sets with different measurement errors. J Magn Reson. 1999;140(1):153-61.

Dunn KJ, LaTorraca GA, Warner JL, et al. On the calculation and interpretation of NMR relaxation time distributions. In: SPE annual technical conference and exhibition. Society of Petroleum Engineers. 1994.

Ge X, Wang H, Fan Y, et al. Joint inversion of $T_{1}-T_{2}$ spectrum combining the iterative truncated singular value decomposition and the parallel particle swarm optimization algorithms. Comput Phys Commun. 2016;198:59-70.

Golub GH, Heath M, Wahba G. Generalized cross-validation as a method for choosing a good ridge parameter. Technometrics. 1979;21(2):215-23.

Guerra V, Hernandez V. Numerical aspects in locating the corner of the L-curve. In: Approximation, optimization and mathematical economics. Heidelberg: Physica-Verlag; 2001. p. 121-31.

Hansen PC. Analysis of discrete ill-posed problems by means of the L-curve. SIAM Rev. 1992;34(4):561-80.

Hansen PC. Rank-deficient and discrete ill-posed problems: numerical aspects of linear inversion. Philadelphia: SIAM; 1998.

Hansen PC, Jensen TK, Rodriguez G. An adaptive pruning algorithm for the discrete L-curve criterion. J Comput Appl Math. 2007;198(2):483-92.

Kaufman L, Neumaier A. PET regularization by envelope guided conjugate gradients. IEEE Trans Med Imaging. 1996;15(3):385-9.

Madsen K, Nielsen HB. Introduction to optimization and data fitting. Lyngby: Technical University of Denmark; 2010.

Morozov VA. On the solution of functional equations by the method of regularization. Sov Math Dokl. 1966;7(1):414-7.

Prammer MG. NMR pore size distributions and permeability at the well site. In: SPE annual technical conference and exhibition. 1994.

Sezginer A. Determining bound and unbound fluid volumes using nuclear magnetic resonance pulse sequences. US Patent 5,363,041, 1994.

Song YQ, Venkataramanan L, Hürlimann MD, et al. $\mathrm{T}_{1}-\mathrm{T}_{2}$ correlation spectra obtained using a fast two-dimensional Laplace inversion. J Magn Reson. 2002;154(2):261-8.

Venkataramanan L, Song YQ, Hürlimann MD. Solving Fredholm integrals of the first kind with tensor product structure in 2 and 2.5 dimensions. IEEE Trans Signal Process. 2002;50(5):1017-26.

Zou YL, Xie RH. A novel method for NMR data compression. Comput Geosci. 2015;19(2):389-401. 